\title{
Fabrication and characterization of $\mathrm{SmCO}_{5} / \mathrm{Nb}$ ferromagnetic/ superconducting hybrid thin films grown by RF magnetron sputtering technique
}

\author{
E. Ongun ${ }^{\text {a }}$, M. Kuru ${ }^{\text {b, a, }}{ }^{*}$, M. Serhatlıoğlu ${ }^{\text {c }}$, M. Hançer ${ }^{\text {d }}$, A.E. Ozmetin ${ }^{\mathrm{e}}$ \\ ${ }^{\text {a }}$ Erciyes University, Department of Materials Science and Engineering, Kayseri, Turkey \\ ${ }^{\mathrm{b}}$ Ondokuz Mayıs University, Department of Materials Science and Engineering, Samsun, Turkey \\ ${ }^{\mathrm{c}}$ Bilkent University, Institute of Materials Science and Nanotechnology (UNAM), Ankara, Turkey \\ ${ }^{\mathrm{d}}$ Muğla Sitkı Koçman University, Metallurgy and Materials Engineering, Muğla, Turkey \\ e Texas A\&M University, Materials Science and Engineering Department, College Station, TX, USA
}

\section{A R T I C L E I N F O}

\section{Article history:}

Received 10 February 2017

Received in revised form

24 July 2017

Accepted 24 July 2017

Available online 25 July 2017

\section{Keywords:}

$\mathrm{Nb} / \mathrm{SmCO}_{5}$ superconductor/ferromagnet

hybrids

Magnetic thin-films

Superconducting thin-films

Photolithography

Superconductivity

\begin{abstract}
A B S T R A C T
Ferromagnet/Superconductor $(\mathrm{F} / \mathrm{S})$ bilayer hybrids show exclusive states due to the mutual interaction between the superconductor and the underlying ferromagnetic substructures in micron scale. In this work, we aimed to observe the effects of the interaction between superconductivity and magnetism, especially the phenomenon involving the orientation and the size of magnetic stripes has been investigated in a coupled ferromagnetic/superconducting thin-film structure. In the proposed $\mathrm{F} / \mathrm{S}$ hybrid system by this work, superconducting niobium thin-films were combined with underlying segments of ferromagnetic $\mathrm{SmCO}_{5}$ substructures. $300 \mathrm{~nm}$ thick magnetic films fabricated by RF magnetron sputtering techniques were topographically grown in patterns with stripes oriented either transverse to or along the direction of current flow. The elemental and microstructural analyses were conducted by EDX, SEM and GIXRD characterization tools. Low-temperature DC transport measurements were conducted by means of four point probe method in a 9T closed-cycle cryogenic refrigeration system. Transport superconducting properties, transition temperature $\mathrm{T}_{\mathrm{C}}(\mathrm{H})$ and second critical field $\mathrm{H}_{\mathrm{C} 2}(\mathrm{~T})$ were measured in a range of applied magnetic field between $\mathrm{H}=0-9 \mathrm{kOe}$ for the hybrid system. The results revealed that the artificial periodic modulation of applied field through preferentially-oriented magnetic stripes could introduce normal and superconducting channels or barriers for the current flow.
\end{abstract}

(C) 2017 Elsevier Ltd. All rights reserved.

\section{Introduction}

In the science and engineering of thin-film materials, the ferromagnetism and superconductivity are introduced as great examples of mutually exclusive states. Within the last decade, the interplay between ferromagnetic and superconducting systems have been studied by experimental groups focusing on transport properties, and also imaging techniques have been employed. When combined in micron scale, the interaction between the stray field from the ferromagnetic substructures and superconducting overlayer leads to unusual superconducting characteristics [1-5].

Several ferromagnetic/superconducting (F/S) hybrid systems

\footnotetext{
* Corresponding author. Ondokuz Mayis University, Department of Materials Science and Engineering, Samsun, Turkey.

E-mail address: kurumehmet54@gmail.com (M. Kuru).
}

were reported in recent years [6-15]. Two comprehensive reviews are also available by Lyuksyutov and Pokrovsky [16], and by the Schuller group [17]. The leading idea is to realize ferromagnetic/ superconducting hybrid systems, where superconducting vortices can interact directly with the stray magnetic fields from the ferromagnetic substructures. As reported by several experiments recently in Refs. [17-19], arrays of magnetic dots or magnetic layers with lattice of holes which were grown on top or under a thin superconducting film could substantially suppress vortex motion close to the critical transition temperature. In Ref. [20], we have searched the superconducting properties of thin PbBi films when combined with thin layers of magnetically-soft Permalloy substructures, which were patterned in an array of $\sim 30 \mu \mathrm{m}$ wide stripes oriented transverse to or along the current path. Based on the results obtained in our previous work, we have in this paper reported an alternative F/S Hybrid system where superconducting Nb thin- 
films (SC-film) were coated over thin layers of magnetically-hard $\mathrm{SmCO}_{5}$ substructures patterned in a finer feature size of $\sim 15 \mu \mathrm{m}$ wide parallel, alternating stripes (FM-stripes) using photolithography technique. Most of the latest investigations on $\mathrm{F} / \mathrm{S}$ systems involve soft magnetic layers or dots, and only a few attempts have been made so far to use highly anisotropic magnetic materials. In addition to all these studies, we have chosen hard magnetic SmCo material which has several advantages. (1) Thin SmCo films can be RF-sputtered on $\mathrm{Si}(100)$ with the easy axis of magnetization aligned perpendicular to the film plane. (2) Because of its high coercivity, thin SmCo films can show a stable magnetization in the field range of the superconducting phase at low temperature.

\section{Structural and electrical modeling}

The F/S hybrid system was designed and realized based on several empirical results obtained after a series of experiments. Each F/S hybrid system was built-up of an array of FM-stripes placed underneath the SC-film in two different orientations, such that; an array of FM-stripes was oriented in parallel, while an array in perpendicular to the direction of current flow ensuring a total spatial coverage of the segment across voltage leads. FM and SC parts were spatially separated by an ultrathin and insulating $\mathrm{Al}_{2} \mathrm{O}_{3}$ capping layer. FM substructures behave as micro flux sources and introduce spatially modulated stray-fields in the SC-film due to relative high permeability of $\mathrm{SmCo}_{5}$ alloy, and the channeling effect through the underlying FM-stripes. Due to relative high permeability of ferromagnetic substructures, the externally applied magnetic field is effectively channeled through the ferromagnetic pattern. This channeling effect continues as the applied field is increased until the particular ferromagnetic material reaches to its saturation magnetization. Using this property, hybrid systems can be designed in such a configuration to form high and low magnetic field regions in the superconducting film. If this field variation is strong enough, parts of the superconductor can be driven normal resulting in a dependence of superconductivity on the current flow direction. Dependent on the stray-field variation, some parts of the SC-film are driven in normal conducting state, while some parts in persistent state [21].

As current flowing in the SC-film is channeled by the stray magnetic field through the long edges of underlying FM-stripes, the current flow path is made up of a parallel connection of intermittent superconducting and resistive alternating ribbons, and so resulting in a current flow over the non-magnetic parts. As current flowing in the SC-film, but transverse to the FM-stripes in this case, the current flow path is then made up of a series connection of superconducting and resistive ribbons, resulting in a voltage drop and an overall resistance arisen across voltage leads of the SC-film. Based on this electrical model, the F/S hybrid system can be designed in order for switching between normal and persistent conducting states by simply rotating the underlying FM-stripes $90^{\circ}$ with respect to the current flow direction [22]. High and low amplitude alternating stray magnetic field distribution in the SCfilm, which is arisen by the preferential orientation of the underlying FM-stripes either transverse to or along the SC-film, is also expected to manipulate the superconducting properties ( $T_{C}$ and $\mathrm{H}_{\mathrm{C} 2}$ ).

The effect of orientation of magnetic stripes upon the superconducting properties; magnetic $\mathrm{SmCO}_{5}$-stripes, grown in "parallel orientation" to the superconducting $\mathrm{Nb}$ overlayer, were polarized by the external magnetic field. The resulting stray fields, located at the stripe edges, caused suppression of superconductivity due to increase in electrical resistance. By this way, easy current channels were formed by the long edges of underlying magnetic stripes. Magnetic $\mathrm{SmCO}_{5}$-stripes, grown in "perpendicular orientation" to the superconducting $\mathrm{Nb}$ overlayer, were polarized by the external magnetic field. The resulting stray fields, located at the stripe edges, caused the suppression of superconductivity due to increase in electrical resistance. By this way, continuous barriers were formed for the transverse current flow through the long edges of underlying magnetic stripes. In order to reveal any numerical facts pertaining to the directional current flow dependence of superconducting properties, a standard 4-point probe transport measurement technique was utilized to perform resistance versus temperature measurements under various $\mathrm{H}$-fields from 0 to $9 \mathrm{kOe}$, and at temperatures in the range of $4-8 \mathrm{~K}$ in a closed-cycle drycryostat.

\section{Experimental}

In this study, RF Magnetron sputtering and resistive thermal evaporation vapor deposition techniques were used along with a series of topographical micro-patterning processes with the aid of various masking tools such as photo-masks and sheet-steel shadow-masks. Fig. 1 introduces the whole fabrication process steps of the proposed F/S Hybrid System starting from the photolithography (PL) stage to the growth of contact pads atop. We aimed to realize a unique bottom-to-top, multi-step, and continuous fabrication process chain to be able to obtain more precise and scalable FM-stripes with lateral feature sizes down to $15 \mu \mathrm{m}$.

Firstly, Si-substrate was spin-coated with positive tone AZ 1518, and soft-baked at $100^{\circ} \mathrm{C}$ on a hot-plate for $50 \mathrm{~s}$. A fine alignment of Si-substrate with photo-mask of acetate film was succeeded using MIDAS MDA-400 M mask aligner in vacuum contact process mode. The geometric pattern on the photo-mask was optically transferred onto the photoresist layer. After pattern transfer, a mixture of bath solution was prepared by diluting AZ 351B developer with deionized water. The micro-patterned resist-mask consists of an array of regularly spaced, parallel, and elongated $15 \mu \mathrm{m}$ wide trenches to function as a mold for the following $\mathrm{SmCo}_{5}$ vapor deposition process. Before introducing into the chamber, argon gas was passed through a custom-made liquid-nitrogen $\left(\mathrm{LN}_{2}\right)$ cold trap station in order to further purify beyond its reported commercial level of $1 \mathrm{ppm} \mathrm{O}_{2}$. Meanwhile, liquid-nitrogen was also charged into the cold-finger reservoir to trap and confine moisture and other impurity gasses remaining inside the chamber. A $2^{\prime \prime}$ diameter highpurity $\mathrm{SmCo}_{5}$ sputtering target was used as deposition source. Following 90 min of pre-sputtering the target, trench openings in the resist-layer were filled-in with sputtered $\mathrm{SmCo}_{5}$ vapor. Thin $\mathrm{SmCo}_{5}$ films were sputter-deposited in $300 \mathrm{~nm}$ thickness onto Sisubstrate at an average deposition rate of $1.0 \AA \mathrm{A} / \mathrm{s}$ under $4.3 \mathrm{mTorr}$ argon partial pressure with a gas flow rate of $15 \mathrm{sccm}$ at room temperature and distance between substrate and target was $10 \mathrm{~cm}$. The RF power density on the surface of $2^{\prime \prime}$ diameter sputtering target was measured approximately $3.95 \mathrm{~W} / \mathrm{cm}^{2}$. Following the growth of $\mathrm{SmCO}_{5}$ thin-films, the samples were soaked in AZ100 remover with ultrasonic agitation to lift-off the remaining resist layer, and to strip-off any residue from the substrate surface. We obtained a thin-film structure with periodic, equally-spaced, and alternating parallel stripes which were finely patterned in micron scale. To prevent magnetic stripes from getting oxidized, the samples were immediately capped with $\mathrm{Al}_{2} \mathrm{O}_{3}$ vapor in $30 \mathrm{~nm}$ thickness by $\mathrm{RF}$ magnetron sputtering technique at room temperature. The samples were then annealed at $500{ }^{\circ} \mathrm{C}$ for $1 \mathrm{~h}$ under partial argon pressure in order to crystallize the as-deposited amorphous magnetic films and to obtain magnetically-hard substructures.

After completion of growth process of FM-stripes, SC-films were grown in $6500 \mu \mathrm{m}$ long, $1000 \mu \mathrm{m}$ wide, and $300 \mathrm{~nm}$ thick dimensions through a set of steel shadow-masks. Each set consisted of two cascaded SC-films placed at $90^{\circ}$ orientation, one was 


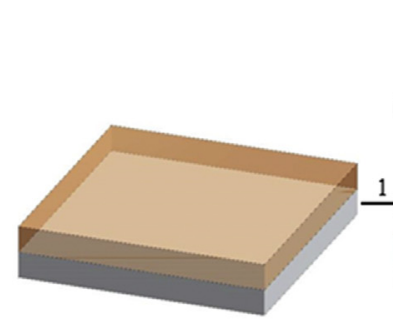

Photoresist spin coated on $\mathrm{Si}$ wafer

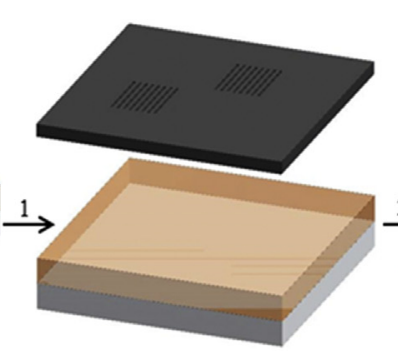

Photomask printed on acetate film

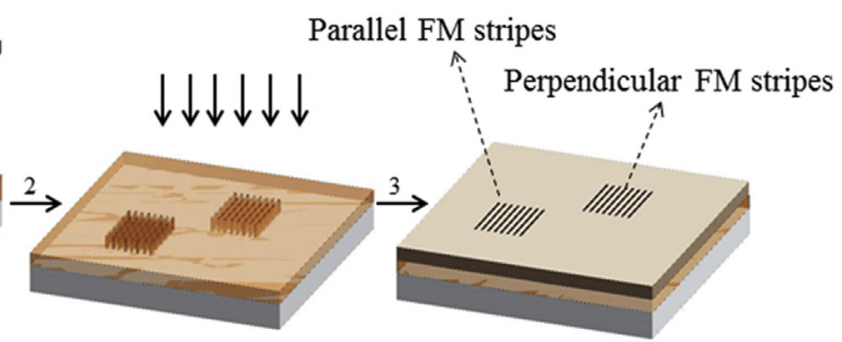

UV light exposed

$\mathrm{SmCo}_{5}$ substructures grown by RF Magnetron sputtering

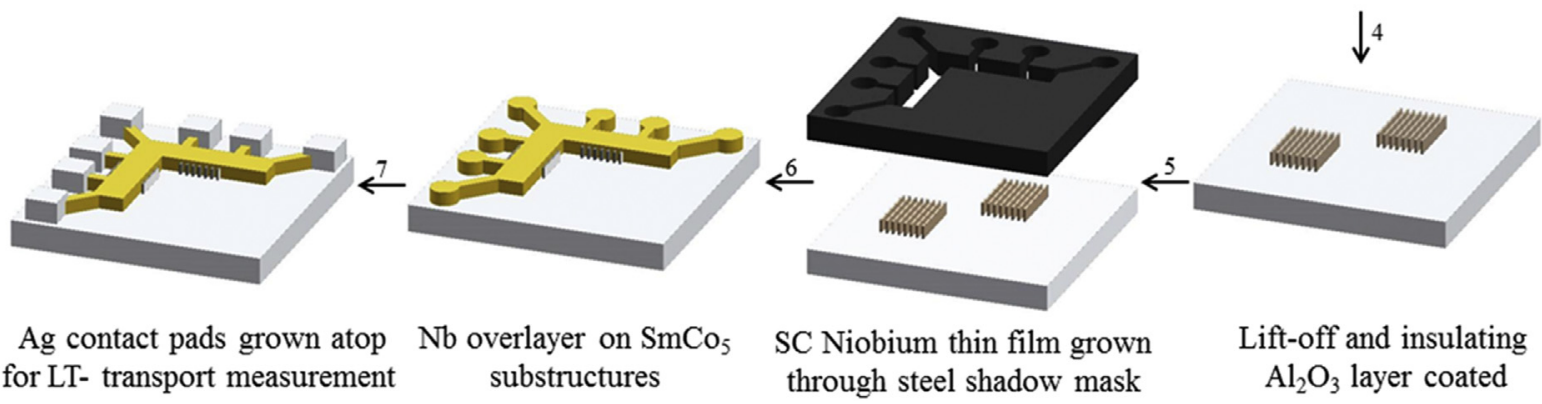

Fig. 1. Complete fabrication process steps of the proposed F/S Hybrid System starting from photolithography stage to the growth of contact pads atop.

dedicated for parallel and the other for perpendicular oriented FMstripes across the voltage leads. This unique design enabled us to fabricate a number of F/S hybrid samples together with a bare SCfilm as reference sample simultaneously under identical vapor deposition conditions. Prior to the start of $\mathrm{Nb}$ deposition process, the vacuum chamber was evacuated down to a base pressure level of $5 \times 10^{-8}$ Torr. A 60 min of continuous pre-sputtering of target was followed by the growth of thin Nb films in $350 \mathrm{~nm}$ thickness at an average deposition rate of $1.12 \AA / s$ at room temperature. Deposition pressure, argon flow rate and RF power density were kept stable at around $4.5 \mathrm{mTorr}, 15 \mathrm{sccm}$ and $4.95 \mathrm{~W} / \mathrm{cm}^{2}$, respectively. Finally, thin $\mathrm{Ag}$ contact padswere grown in $40 \mathrm{~nm}$ thickness by resistive thermal-evaporation technique using a steel shadowmask.

\section{Characterization}

\subsection{Structural characterization}

The thickness, microstructural and surface morphological analyses of $\mathrm{SmCO}_{5}$ and $\mathrm{Nb}$ films were examined with Zeiss EVO LS10 (SEM). The elemental composition was measured by energy dispersive analysis (EDX). Morphological investigation plays an important role in determining the surface characteristics and the nature of the films. Surface morphology of the $\mathrm{SmCo}_{5}$ films deposited with RF magnetron sputtering was investigated by SEM. Fig. 2 (a) and (b) shows both surface and cross-section SEM images of the $\mathrm{SmCO}_{5}$ and $\mathrm{Nb}$ films, respectively. These images show that substrate is fully covered with nano-sized $\mathrm{SmCo}_{5}$ and $\mathrm{Nb}$ grains.

EDX analyses of $\mathrm{SmCO}_{5}$ and $\mathrm{Nb}$ thin-films are shown in Fig. 2 (c), and (d), respectively. An EDX analysis of $\mathrm{SmCo}_{5}$ thin-film revealed an elemental composition of 17.20 at \%Sm and 82.80 at \%Co indicating a stoichiometric $\mathrm{SmCO}_{5}$ alloy composition, and an EDX analysis of $\mathrm{Nb}$ film revealed an elemental composition of about 100 at \% Nb.

In Fig. 3, a surface top-view of lithographically grown $\mathrm{SmCO}_{5}$ thin-film exposes $\sim 13.5 \mu \mathrm{m}$ wide stripes and inset of Fig. 3 , the distance between two neighbouring magnetic stripes is $\sim 25 \mu \mathrm{m}$. As seen in Fig. 3, the magnetic stripes are properly located on the surface and these stripes have a fairly uniform and dense morphology.

Grazing incidence X-ray diffraction (GIXRD) tool with $\mathrm{Cu} / \mathrm{K}_{\alpha}$ radiation was employed in order for phase characterization of $\mathrm{SmCO}_{5}$ deposits to reveal the crystal structures formed. Fig. 4 shows a typical XRD pattern belonging to $\mathrm{SmCo}_{5}$ film annealed at $500{ }^{\circ} \mathrm{C}$ for $1 \mathrm{~h}$ under argon atmosphere. Crystallization of $\mathrm{SmCo}_{5}$ thin films can be seen clearly in the XRD spectrum given in Fig. 4. The $\mathrm{SmCo}_{5}$ phase with the $\mathrm{CaCu}_{5}$ structure was observed when annealed at $500{ }^{\circ} \mathrm{C}$. Diffraction peaks corresponding to the crystalline phases of $\mathrm{SmCo}_{5}$ alloy have been observed in the pattern. The average crystallite size (D) of the structures was calculated from the peak full width at the half maximum (FWHM) of a peak ( $\beta$ ), using the DebyeScherrer's equation [23].

$D=\frac{0.9 \lambda}{\beta \cos \theta}$

where $\lambda$ is the wavelength of $X$-ray radiation, $\theta$ is the Bragg's angle of the peaks and $\beta$ is the angular width of peaks at FWHM. Fig. 4 shows an XRD pattern of the $\mathrm{SmCO}_{5}$ film diffraction main peak from (110) of $\mathrm{SmCo}_{5}$ which is clearly observed at around $43.58^{\circ}$ for 20. Also, phase of $\mathrm{SmCo}_{7}$ (107) is seen in the X-ray diffraction pattern. When SmCo films were subjected to heat treatment; the crystal structure of SmCo phase was formed in hexagonal $\mathrm{CaCu}$ -type) structure. The structure of $\mathrm{SmCo}_{7}$ phase can be generated from that of SmCo by an ordered substitution of Co dumb-bells into some of the Sm sites. Consequently, these two phases are crystallographically coherent, and particularly have the same easymagnetization axis. The mean crystallite size of the structure is $16.31 \mathrm{~nm}$ which consistent based on ASTM E112-96 standard.

\subsection{Low-temperature transport characterization}

Fig. 5 shows a photograph belonging to a set of cascaded $\mathrm{F} / \mathrm{S}$ hybrid samples which were wire-bonded on a custom-designed sample puck, and mounted on the VTI head for low temperature 


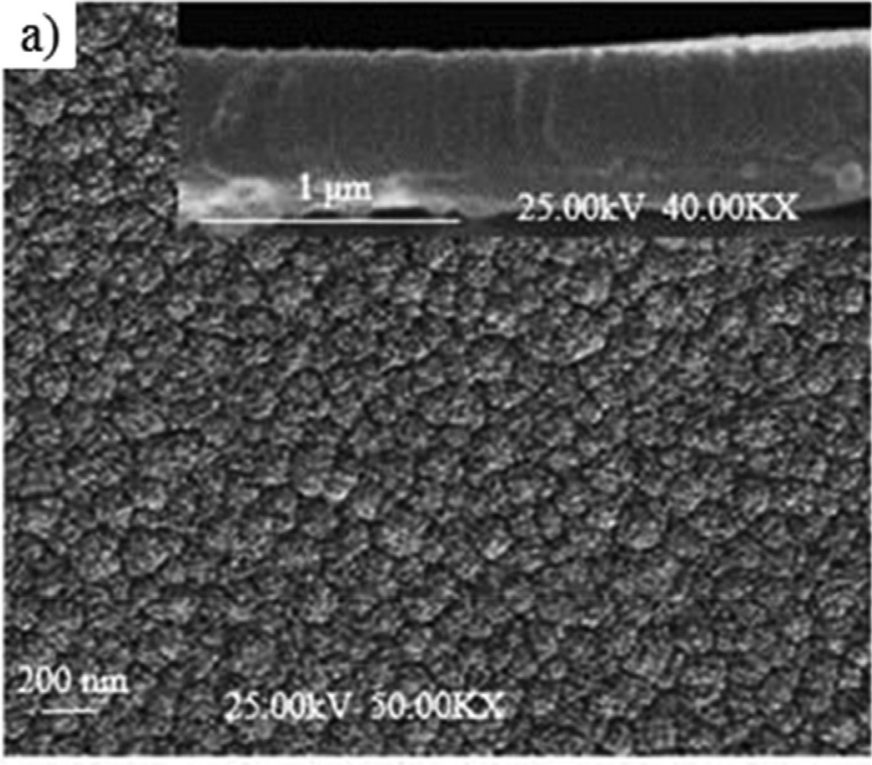

c)

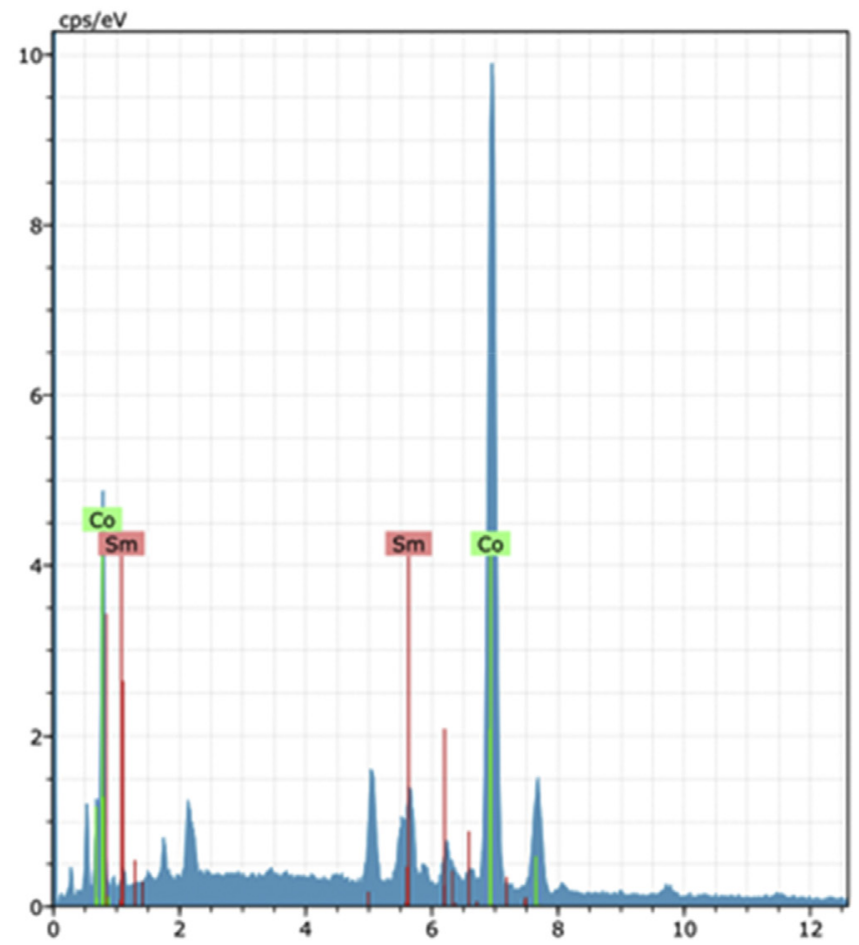

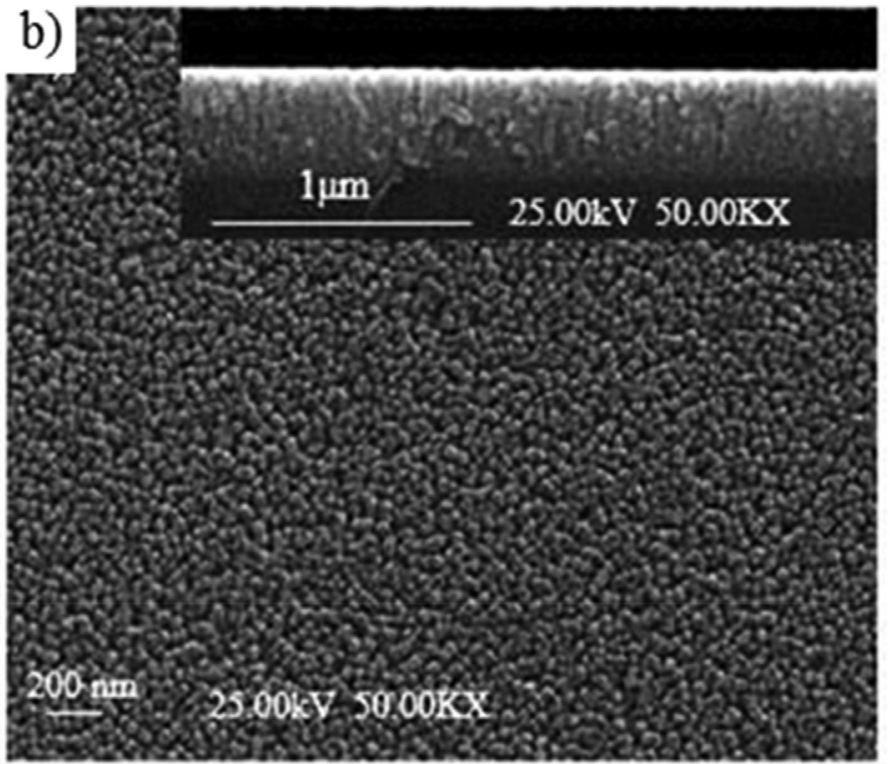

d)

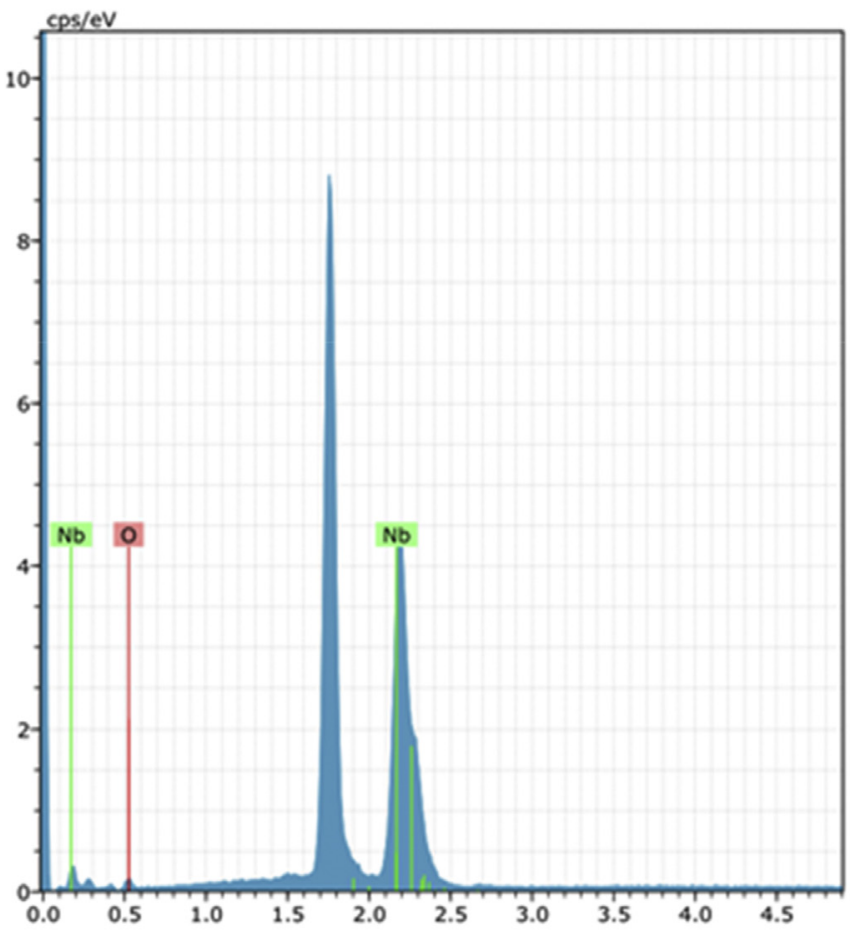

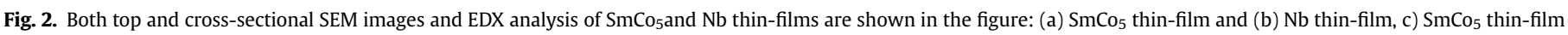
composed of 82.80 at \%Co and 17.20 at \%Sm d) Nb thin-film composed of about 100 at \%Nb.

dc transport measurements. In this sample set, one hybrid film was made of a SC-film with underlying parallel FM-stripes, and the other hybrid film was made of a SC-film with underlying perpendicular FM-stripes.

In order to reveal the directional current flow dependence of superconducting properties with respect to the orientation of underlying FM-stripes, we conducted the low-temperature transport measurements of each $\mathrm{F} / \mathrm{S}$ hybrid thin-film sample. To determine the critical temperature $\left(\mathrm{T}_{\mathrm{C}}\right)$ values; a constant dc current of $100 \mu \mathrm{A}$ was passed through the superconducting $\mathrm{Nb}$-film, and voltage drops were measured from $\mathrm{T}>\mathrm{T}_{\mathrm{C}}$ to $\mathrm{T}<\mathrm{T}_{\mathrm{C}}$ in the range of $8 \mathrm{~K}-4 \mathrm{~K}$ while the samples were swept from 0 to 9 kOe.

\subsubsection{Bare SC-film}

Fig. 6a shows resistance vs. temperature (R-T) curves, normalized to the residual resistance value measured at $8.0 \mathrm{~K}$ for a bare SCfilm reference sample under $\mathrm{H}$-fields from 0 to 9 kOe. Superconducting transition onset temperature $\left(\mathrm{T}_{\mathrm{C}}\right)$ occurred at $7.6 \mathrm{~K}$ under zero-field.

The R-T curves of the sputter-deposited niobium thin-film sample (SC-film) in Fig. 6a were used as reference data to investigate and reveal the superconducting properties and the directional 


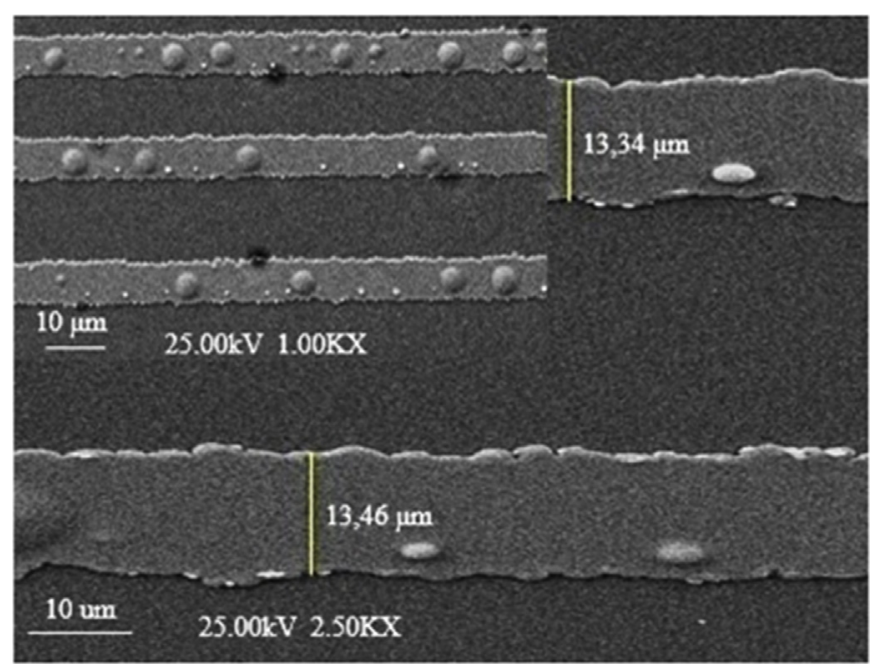

Fig. 3. A surface top-view of lithographically grown $\mathrm{SmCo}_{5}$ thin-film exposes $\sim 13.5 \mu \mathrm{m}$ wide stripes.

current flow dependence when combined in contact junction with ferromagnetic samarium-cobalt thin-film stripes (FM-stripes) in two different orientations, either parallel or perpendicular to the current flow direction, separately.

\subsubsection{Parallel F/S hybrid}

Fig. $6 \mathrm{~b}$ shows resistance vs. temperature curves, normalized to the residual resistance value measured at $8.0 \mathrm{~K}$ for a SC-film with parallel $\mathrm{FM}$-stripes under $\mathrm{H}$-fields from 0 to $9 \mathrm{kOe}$. In this configuration, current flows in the SC-film through the long edges of underlying FM-stripes across voltage-leads. Superconducting transition onset temperature $\left(\mathrm{T}_{\mathrm{C}}\right)$ occurred at about $7.75 \mathrm{~K}$ under zero-field. The relative difference between the R-T curves of parallel F/S hybrid sample and the bare SC-film sample becomes larger at

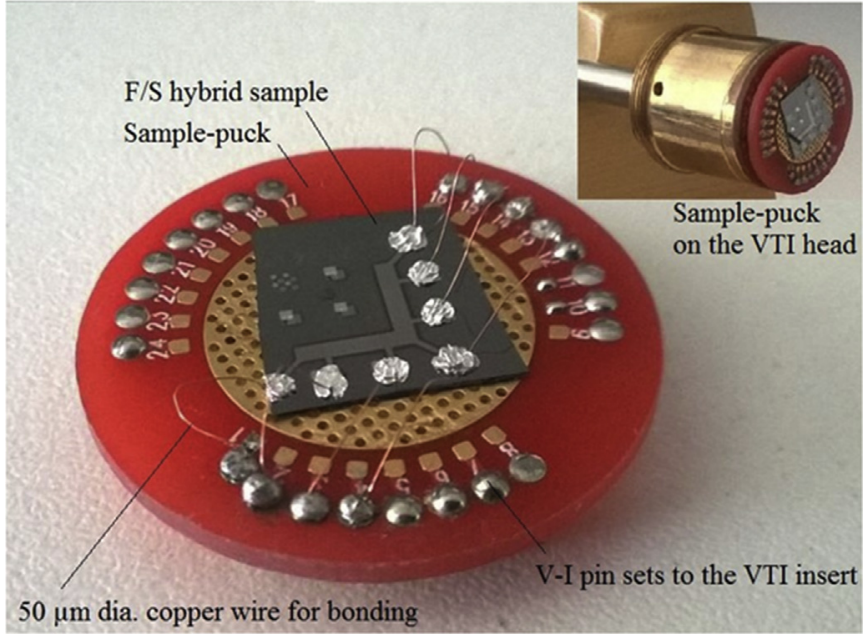

Fig. 5. A photograph belongs to a set of FM/SC hybrid samples wire-bonded on a custom-designed sample puck.

lower temperatures. Stray magnetic fields, generated from the long edges of underlying FM-stripes, penetrate the SC-film and result in easy current channels across. As a result of this interaction, superconducting transition onset values of parallel $\mathrm{F} / \mathrm{S}$ hybrid sample are slightly shifted to higher temperature values.

\subsubsection{Perpendicular $F / S$ hybrid}

Fig. 6c shows resistance vs. temperature curves, normalized to the residual resistance value measured at $8.0 \mathrm{~K}$ for a SC-film with perpendicular FM-stripes under $\mathrm{H}$-fields applied from 0 to $9 \mathrm{kOe}$. In this configuration, current flows in the SC-film transverse to the long edges of underlying FM-stripes resulting in a voltage drop across voltage-leads. A different result was obtained when FMstripes were oriented perpendicular to SC-film. Onset values were shifted to lower temperatures with respect to that of the bare and

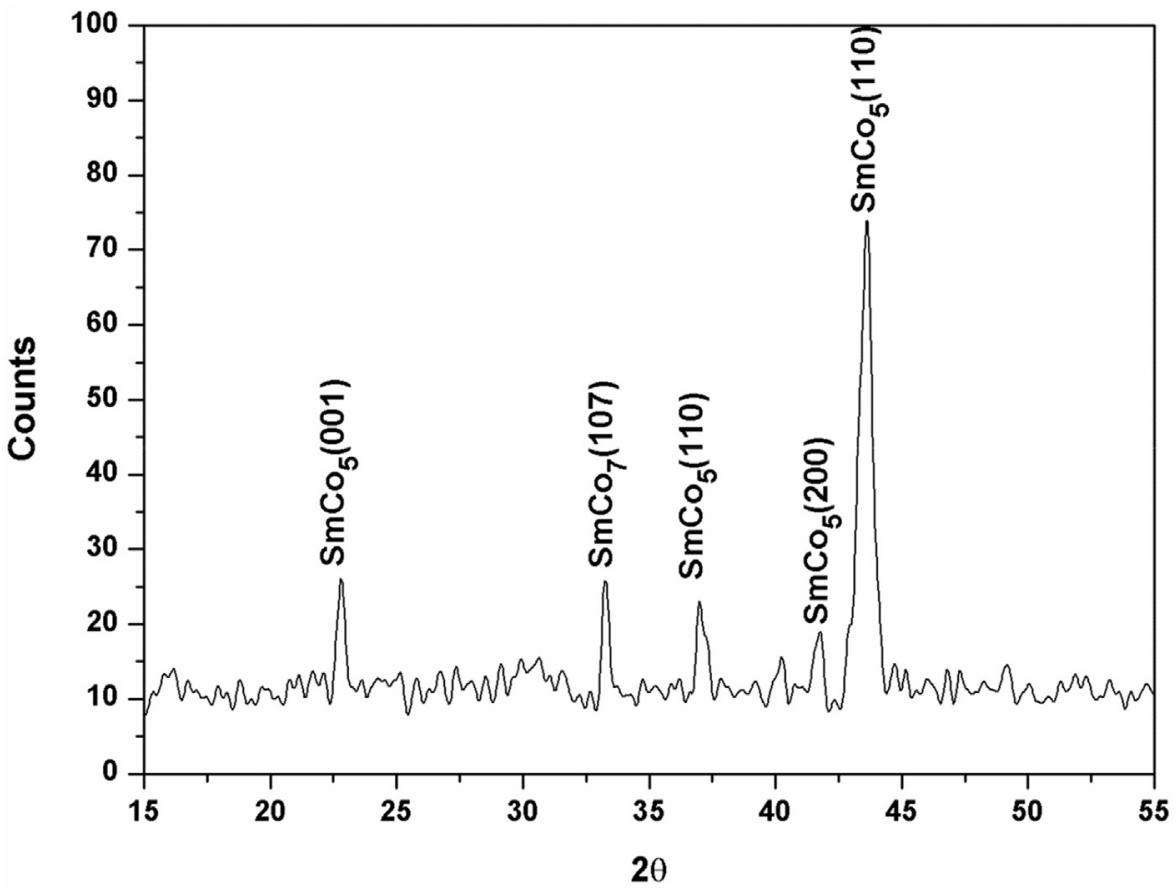

Fig. 4. X-ray diffraction analysis of sputter-deposited $\mathrm{SmCo}_{5}$ thin-film sample which was annealed at $500{ }^{\circ} \mathrm{C}$ for $1 \mathrm{~h}$ under argon atmosphere. 

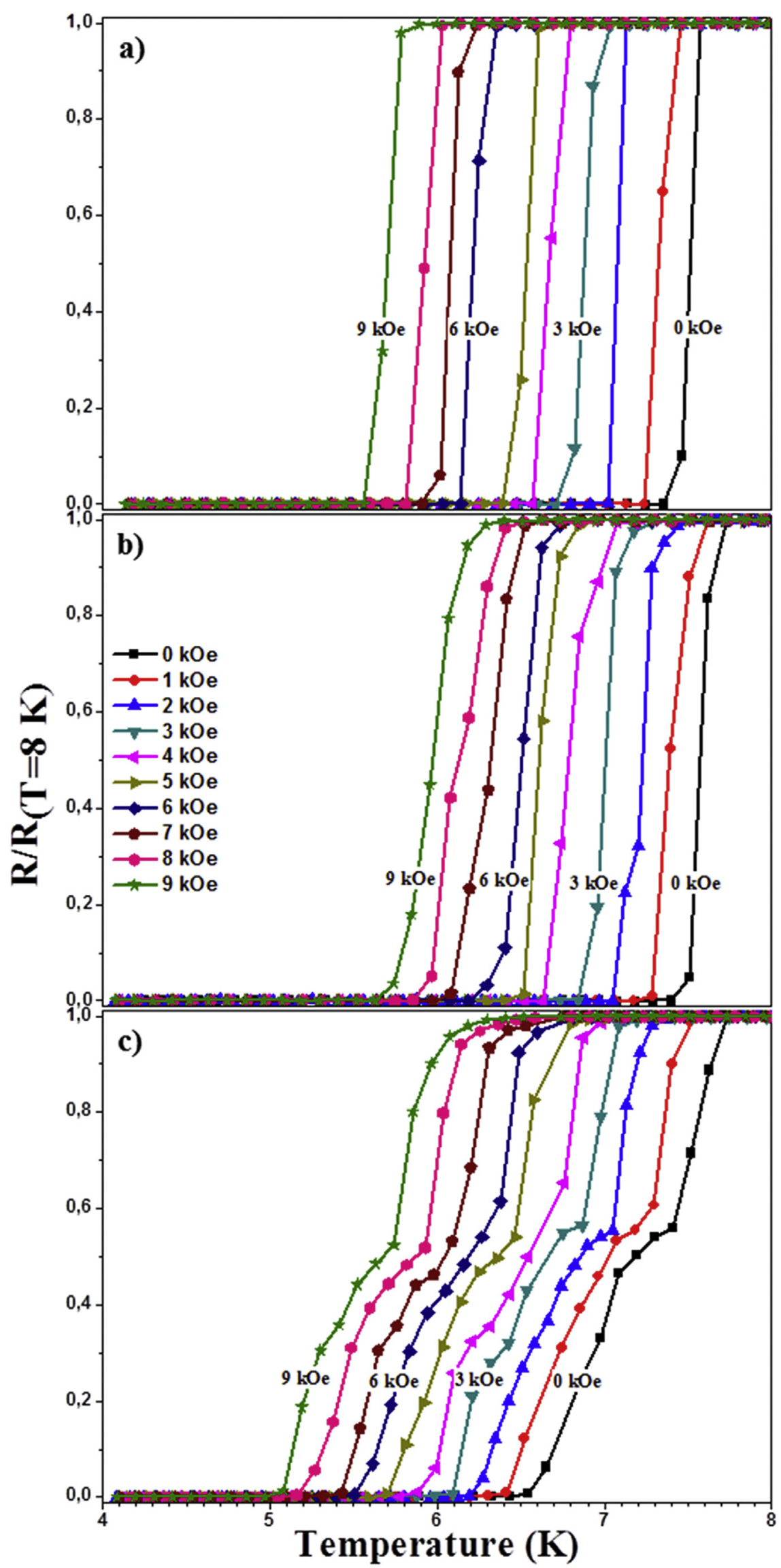

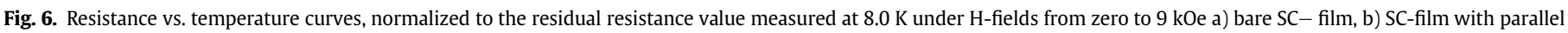
FM-stripes, c)SC-film with perpendicular FM-stripes. 
the parallel F/S hybrid samples.

Each normalized resistance curve $\mathrm{R}(\mathrm{T})$ has revealed an unusual kink formation followed by an anomalous widening when fell to about $50 \%$ of its residual resistance value. This observation indicates an inhomogeneous transition regime mixed of both superconducting and normal conducting states simultaneously. Stray fields, located at the long edges of perpendicular oriented magnetic stripes, caused suppression of superconductivity and increase of resistance across voltage-leads by forming continuous barriers along the superconducting film.

The stray magnetic fields, generated from the underlying ferromagnetic $\mathrm{SmCo}_{5}$ stripes, are expected to effect and change the second-critical field $\left(\mathrm{H}_{\mathrm{C} 2}\right)$ values of superconducting $\mathrm{Nb}$ films dependent on the current flow direction with respect to the orientation of stripes, and also of the bare $\mathrm{Nb}$ control sample with no stripe underneath.

Fig. 7 reveals this effect which is arisen due to the mutual interaction between ferromagnetic and superconducting parts combined in micro scale. In the figure, a set of second-critical field $\left(\mathrm{H}_{\mathrm{C} 2}\right)$ values were plotted for each sample; parallel $\mathrm{F} / \mathrm{S}$ hybrid, perpendicular F/S hybrid, and the bare control sample on the same graph.

It was revealed in Fig. 7 above that the result obtained by this work is also consistent with $\mathrm{R}(\mathrm{T})$ behavior as it has been already reported in Ref. [4] for identical hybrid geometry made up of Fe as ferromagnetic constituent material, where an improvement in the second-critical field values $\left(\mathrm{H}_{\mathrm{C} 2}\right)$ was observed by the authors.

Indeed, the resultant stray magnetic field in the superconducting film above the non-magnetic region is lower than the applied magnetic field, while the resultant stray magnetic field in the superconducting film above the magnetic region $\left(\mathrm{SmCO}_{5}\right.$ stripes) is higher than the applied magnetic field. Another effect, arisen due to the mutual interaction between FM and SC parts, is the inhomogeneous field distribution across the ferromagnetic stripes. Since $\mathrm{H}_{\mathrm{C} 2}$ increases as $\mathrm{T}$ decreases, a larger portion of the film over the non-magnetic regions becomes superconducting, while still a smaller portion of the superconducting film over the
$\mathrm{SmCO}_{5}$ film is driven normal by the field. These two effects can explain the differences between the measured $\mathrm{H}_{\mathrm{C} 2}$ for current flow parallel to the magnetic stripes compared to that of the control film, as shown in Fig. 7 above.

\subsection{The persistent/normal transition regions}

When the ferromagnet-superconductor hybrid structure is placed in a constant homogeneous external magnetic field $(\mathrm{H})$, the magnetic field is amplified and redistributed over the SC film due to the high magnetic permeability of the underlying ferromagnetic stripes. This leads to the creation of alternating parallel regions of low and high magnetic fields. The local magnetic field induced in regions of SC film directly above the magnetic stripes can be high enough to exceed the second critical field $\left(\mathrm{H}_{\mathrm{C} 2}\right)$, and thus, superconductivity in this portion of the film is suppressed (in normal/ resistive state). On the other hand, the local magnetic field passing through the parts of SC film directly above the nonmagnetic portions between neighboring magnetic stripes is still well below $\mathrm{H}_{\mathrm{C} 2}$, and thus, these parts of film remain SC (in persistent state). As a result of our observation, there could be two possible configurations depending on the relative orientation between the ferromagnetic stripes and the current flow. In the case of current flowing parallel to the underlying magnetic stripes, the flow path is made up of a parallel network of SC and resistive ribbons, and so the current flows over the SC regions. In the case of current flowing perpendicular to the underlying magnetic stripes, the path is made up of a series connection of SC and resistive ribbons, and so the current experiences an overall resistance, and a voltage drop is generated between the two ends. Therefore, this directional current flow dependence naturally forms either the "normal" or the "persistent" state in the FM/SC hybrid structure. By changing the direction of the current flow relative to the orientation of the magnetic stripes, the structure can be easily switched between normal and persistent states. This could be achieved by mechanically rotating the underlying ferromagnetic stripes $90^{\circ}$ with respect to the SC film with the use of a rotary $x-y-z$ translation stage.

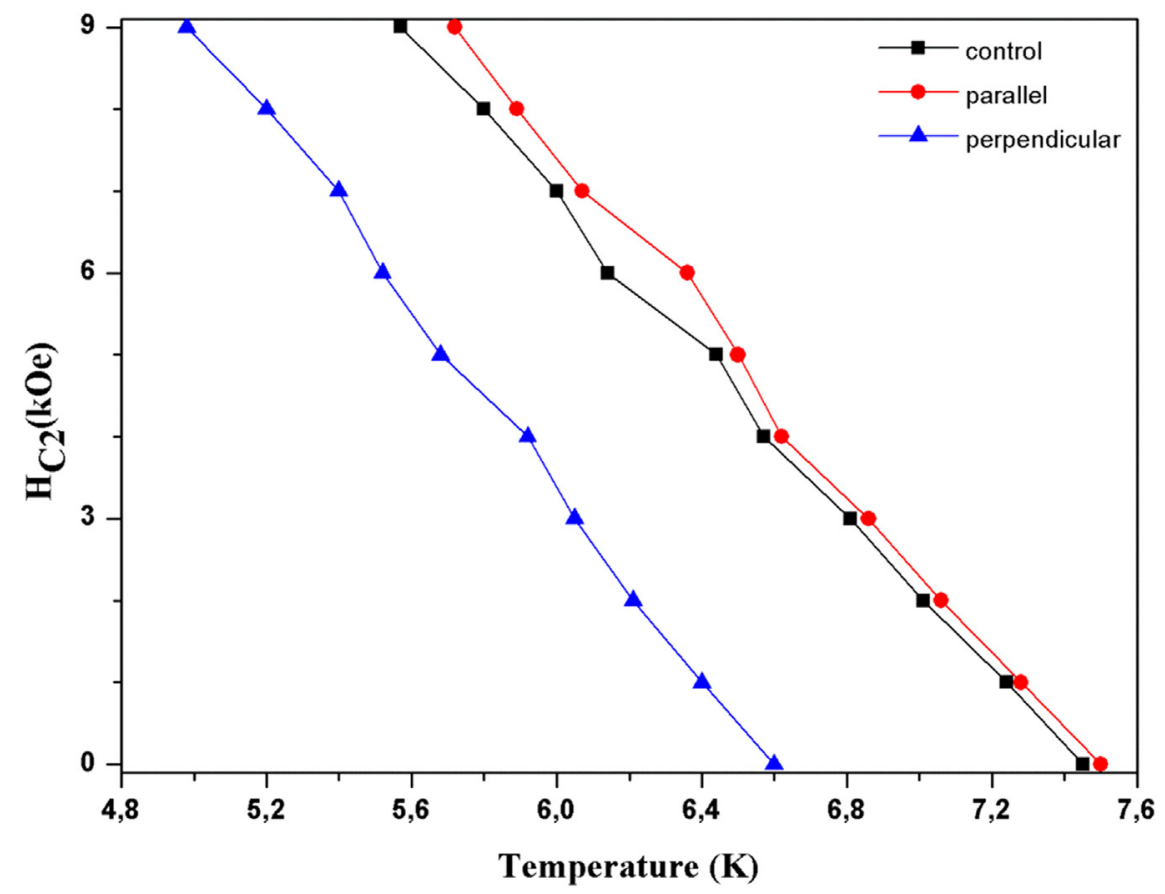

Fig. 7. A set of second-critical field $\left(\mathrm{H}_{\mathrm{C} 2}\right)$ values were plotted for each sample; parallel $\mathrm{F} / \mathrm{S}$ hybrid, perpendicular $\mathrm{F} / \mathrm{S}$ hybrid, and the bare control sample. 
Initially the magnetic stripes are aligned parallel to the current flow direction and so staying in the persistent state. To switch to the normal state, the magnetic stripes are rotated $90^{\circ}$ with respect to the SC film. In this work, two identical samples of the proposed F/S hybrid system, which were composed of FM-stripe patterns one grown in parallel and the other in perpendicular orientation to current flow direction, were cooled below the onset temperature of superconducting transition region. Both samples were biased with a dc current rated at $100 \mu \mathrm{A}$, and swept under various $\mathrm{H}$-fields from zero to $3 \mathrm{kOe}$. The normalized resistance vs. temperature curves were plotted using the collected I-V data for each field. Fig. 8 shows both persistent and normal conducting states of two identical samples on the same graph. The stray magnetic field, localized at the long edges of FM-stripes, determines the conducting state of each sample dependent on the orientation of stripes with respect to the current flow direction.

In Fig. 8, the normalized $\mathrm{R}(\mathrm{T})$ curves of parallel and perpendicular F/S hybrid samples were plotted under zero-field, on which the persistent and normal transition regions were observed to occur at around $7.3 \mathrm{~K} \pm 0.1 \mathrm{~K}$, hatched in the figure. At $7.3 \mathrm{~K} \pm 0.1 \mathrm{~K}$; parallel $\mathrm{F} / \mathrm{S}$ hybrid sample shows zero resistance as being in persistent state, while perpendicular F/S hybrid sample shows resistance of about $60 \%$ of its residual value as being in resistive normal state.

\section{Conclusions}

Due to the stray magnetic fields, which were generated from the long edges of underlying ferromagnetic (FM) $\mathrm{SmCo}_{5}$ stripes, intermittent superconducting and normal conducting parts were formed across the superconducting (SC) Nb over layer. Depending on the current flow direction relative to the orientation of FMstripes, the superconducting and normal conducting regions are naturally configured into either parallel or series connections of persistent and normal conduction paths.

This work introduces growth and characterization of a prototype $\mathrm{F} / \mathrm{S}$ hybrid system to operate as a persistent/normal current states based on the directional current flow dependence. In parallel-contact switching mode; easy current channels are formed across voltage-leads in the SC-film due to the stray-field localization at the long edges of FM-stripes, while in perpendicular-contact switching mode; continuous resistive barriers are formed for the transverse current flow to the FM-stripes. A temperature zone, through which either conducting states occur dependent on the orientation of FM-stripes, could be precisely specified for a persistent/normal current switch operation. The topographical effects resulting from surface corrugation of underlying ferromagnetic substructures could also be an interesting topic of research to investigate if any enhancement occurs at the superconducting
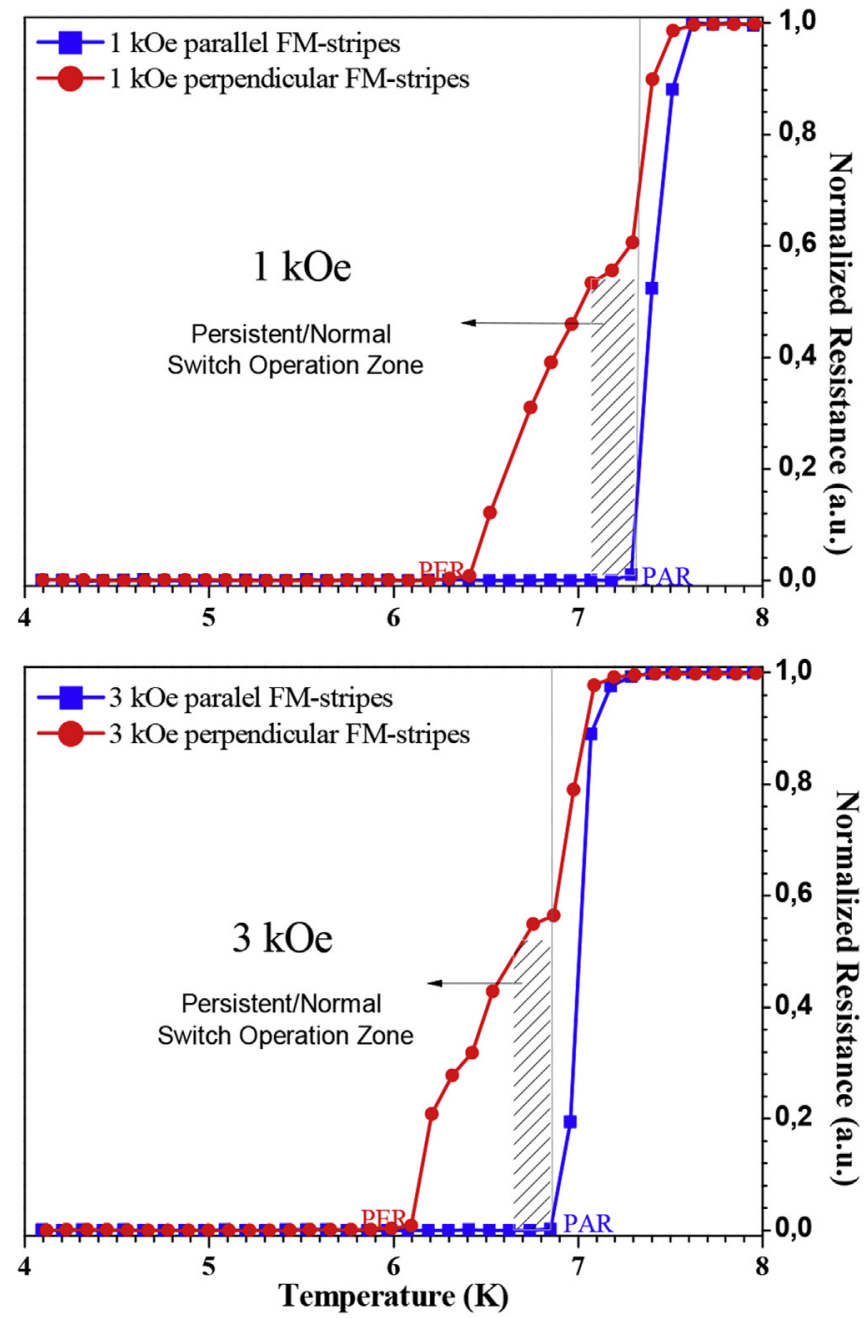

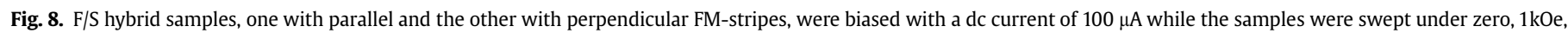
2 kOe, and 3 kOe fields. Persistent/normal transitions were defined dependent on the orientation of FM-stripes as shown hatched in the R-T curves above. 
properties.

\section{Acknowledgement}

The authors acknowledge use of the photolithography facility of ERNAM-Nanotechnology Research Center at Erciyes University.

\section{References}

[1] A.E. Ozmetin, M.K. Yapici, J. Zou, I.F. Lyuksyutov, D.G. Naugle, Micromagnetsuperconducting hybrid structures with directional current flow dependence for persistent current switching, Appl. Phys. Lett. 95 (022506) (2009).

[2] A.E. Ozmetin, E. Yazici, K. Kim, K.D.D. Rathnayaka, I.F. Lyuksyutov, D.G. Naugle, Hysteresis of the phase diagram in the ferromagnet-superconductor hybrids, Int. J. Mod. Phys. B 27 (No.15) (2013).

[3] A.E. Ozmetin, A Method for Simulating the Superconducting Properties in Ferromagnetic-superconducting Hybrid Systems, The 29th Int. Review of Progress in Applied Computational Electromagnetics, Monterey, CA, Vol. Modelling and Simulation in Electromagnetic Engineering, ACES, 2013.

[4] A.E. Ozmetin, K.D.D. Rathnayaka, D.G. Naugle, I.F. Lyuksyutov, Strong increase in critical field and current in magnet-superconductor hybrids, J. Appl. Phys. 105 (2009) 07E324.

[5] I.F. Lyuksyutov, D.G. Naugle, A.E. Ozmetin, M.K. Yapici, J. Zou, Vortex pinning by an inhomogeneous magnetic field, J. Supercond. Nov. Magn 23 (6) (2010) 1079-1082.

[6] S. Erdin, I.F. Lyuksyutov, V.L. Pokrovsky, V.M. Vinokur, Topological textures in a ferromagnet-superconductor bilayer, Phys. Rev. Lett. 88 (017001) (2001).

[7] S. Erdin, A.F. Kayali, I.F. Lyuksyutov, V.L. Pokrovsky, Interaction of mesoscopic magnetic textures with superconductors, Phys. Rev. B 66 (2002) 014414.

[8] L.N. Bulaevskii, E.M. Chudnovsky, M.P. Maley, Magnetic pinning in superconductor-ferromagnet multilayers, Appl. Phys. Lett. 76 (2000) 2594.

[9] Z. Yang, M. Lange, A. Volodin, R. Szymczak, V.V. Moshchalkov, Domain-wall superconductivity in superconductor-ferromagnet hybrids, Nat. Mater 3 (2004) 793-798

[10] M.Z. Cieplak, X. Cheng, C.L. Chien, H. Sang, Origin of pinning enhancement in a ferromagnet-superconductor bilayer, J. Appl. Phys. 97 (026105) (2005).
[11] V. Vlasko-Vlasov, U. Welp, G. Karapetrov, V. Novosad, D. Rosenmann, M. Iavarone, A. Belkin, W.-K. Kwok, Guiding superconducting vortices with magnetic domain walls, Phys. Rev. B 77 (2008) 134518.

[12] L.Y. Zhu, T.Y. Chen, C.L. Chien, Altering the superconductor transition temperature by domain-wall arrangements in hybrid ferromagnet-superconductor structures, Phys. Rev. Lett. 101 (017004) (2008).

[13] A.I. Buzdin, Proximity effects in superconductor-ferromagnet heterostructures, Rev. Mod. Phys. 77 (2005) 935-976.

[14] I.F. Lyuksyutov, D.G. Naugle, Magnetic nanorods/superconductor hybrids, Int J. Mod. Phys. B 17 (2003) 3713.

[15] I.F. Lyuksyutov, D.G. Naugle, Magnet/superconductor nanostructures, Int. J. Mod. Phys. B 17 (3441) (2003).

[16] I.F. Lyuksyutov, V.L. Pokrovsky, Ferromagnet-superconductor hybrids, Adv.in Phys. 54 (No.1) (2005) 67-136.

[17] M. Velez, J.I. Martin, J.E. Villegas, A. Hoffmann, E.M. Gonzalez, J.L. Vicent, I.K. Schuller, Superconducting vortex pinning with artificial magnetic nanostructures, J. Magn. Magn. Mater 320 (No. 21) (2008) 2547-2562.

[18] J.C. Lodder, Patterned nanomagnetic films, in: R. Skomski, D. Sellmyer (Eds.), Advanced Magnetic Nanostructures, Springer, US, 2006, pp. 261-293 (Chapter 10$)$.

[19] M. Kustov, P. Laczkowski, D. Hykel, K. Hasselbach, F. Dumas-Bouchiat, D. O’Brien, P. Kauffmann, R. Grechishkin, D. Givord, G. Reyne, O. Cugat, and N. M Dempsey, "Magnetic characterization of micropatterned $\mathrm{Nd}-\mathrm{Fe}-\mathrm{B}$ hard magnetic films using scanning Hall probe microscopy". Journal of Applied Physics 108, 0639142010.

[20] A.E.Ozmetin, E.Ongun, M.Kuru, and E.Yazıcı, "Fabrication and characterization of ferromagnetic-superconducting hybrid films grown by combined PVD techniques", Proceedings of the Science and Applications of Thin Films Conference (SATF2014), Çeşme-İzmir, 15-19 Sep 2014, Applied Surface Science Vol. 350, pp. 2-5, 2015.

[21] E. Ongun, Low Temperature Electrical and Magnetic Characterization of Superconducting/Ferromagnetic Thin-films Deposited by Various Physical Vapor Deposition Techniques, PhD Dissertation, Erciyes University Joint Doctoral Program, 2016.

[22] V.K. Vlasko-Vlasov, E. Palacious, D. Rosenmann, J. Pearson, et al., Self-healing patterns in ferromagnetic-superconducting hybrids, Supercond. Sci. Technol. 28 (No. 3) (2015), 035006 (8pp).

[23] B.D. Cullity, C.D. Graham, Introduction to Magnetic Materials, Wiley, 2009. 